See discussions, stats, and author profiles for this publication at: https://www.researchgate.net/publication/353428733

\title{
Historical Data Trend Analysis in Extended Reality Education Field
}

Conference Paper · May 2021

DOI: 10.1109//CVR51878.2021.9483828

\section{CITATION}

1

6 authors, including:

2. Yongkang Xing

Guangdong University of Finance

9 PUBLICATIONS 10 CITATIONS

SEE PROFILE

2. Conor Fahy

De Montfort University

13 PUBLICATIONS 81 CITATIONS

SEE PROFILE

Some of the authors of this publication are also working on these related projects:

FuzzyPhoto View project

Project Audiences of the Future View project

\section{READS}

62

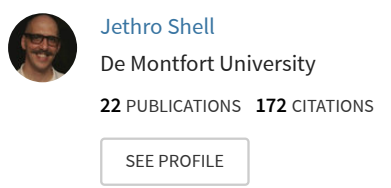




\section{Historical Data Trend Analysis in Extended Reality Education Field}

\author{
Yongkang Xing \\ Institute of Artificial Intelligence \\ De Montfort University \\ Leicester, United Kingdom \\ P2576780@my365.dmu.ac.uk
}

\author{
Conor Fahy \\ Institute of Artificial Intelligence \\ De Montfort University \\ Leicester, United Kingdom \\ conor.fahy@dmu.ac.uk
}

\author{
Zhanti Liang \\ Department of Art Design and Creative \\ Industry \\ Nanfang College Guangzhou \\ Guangzhou, China \\ 1220639541@qq.com
}

\author{
Kexin Guan \\ Operation Department \\ Jiangxi Tanwan Information Technology \\ Co., Ltd \\ Guangzhou, China \\ 243132397@qq.com
}

\author{
Jethro Shell* \\ Institute of Artificial Intelligence \\ De Montfort University \\ Leicester, United Kingdom \\ jethros@dmu.ac.uk
}

\author{
Bingnan Liu \\ Center of Experimental Teaching \\ Guangdong University of Finance \\ Guangzhou, China \\ 70-177@gduf.edu.cn
}

\begin{abstract}
The arrival of the digital age brings Virtual Reality, Augmented Reality, and Mixed Reality technologies into our daily life. It provides a brand-new user experience to composite with real environments. Due to the development of related devices in recent years, the highly interactive connections between users and devices have gradually evolved. The paper starts from a literature review to discuss Virtual Reality, Augmented Reality, and Mixed Reality's history and social impact. The review reveals not only the traditional historical review but also contains a data research study. The research focuses on the case study paper, which proposed a bright, interactive future with technology in educational field. We compared the proposed future view and the current development. This paper collected 269 citations from 2005 to 2020 and analyzed them, assessing whether they belonged to technical or theoretical paper. The paper uses the collected data to discuss industrial developing trends and indicates the possible future view based on the data study result.
\end{abstract}

Keywords-virtual reality, argument reality, mixed reality, literature review, history, citation

\section{INTRODUCTION}

The arrival of the digital age brings Extended Reality (XR) into our daily life. CCS-Insight reports that the value of XR devices sold is expected to increase from US $\$ 1.5$ billion in 2017 to US $\$ 9.1$ billion by 2021 [1]. The XR technologies provide brand-new Information and Communication Technologies (ICTs) to affect the user experience in interacting with the environment [1]. The technology does not only open a new horizon in the game industry but also shows huge potential in the educational, commercial, and medical fields. It generates optimal customer experiences with the combination of virtual-physical touchpoint. Considering the above market value and developing potential, it is necessary to discuss XR's concept and its characteristics. In fact, XR is a combination concept with Virtual Reality, Augmented Reality, and Mixed Reality [2]. In the following sections, we review the concept of
Virtual Reality, Argument Reality and Mixed Reality. Each section critically analyzes the classified Reality's definition, inconsistencies, advantages and weaknesses.

\section{A. Virtual Reality}

Virtual Reality (VR) is a technical field that users can operate computers and interact with the Virtual Environment (VE) [2]. VR has three major characteristics: 360-degree experience, real-time and interactive. Speicher concludes that 360-degree experience is the key feature of VR [3]. 360-degree experience does not just show the number of degrees, it means that the device can provide an immersive experience in the VE. Moreover, users will be largely immersed into the VE in the visualization and motion aspects. The second feature is the real-time criteria. Each behavior of the 3D entities should run in real-time so that the users will be immersed in a pseudonatural manner [2]. The third feature is interaction. The system should provide the functionalities for users to act on the behavior in VE such as moving around and manipulating [2].

VR has many bright prospects in some fields such as construction, manufacture, design, medical and learning area. VR can use its immersive features to improve communication efficiency and provide visual education to better understand certain complex phenomena [2]. However, VR has an inevitable weakness that it can generate some sicknesses such as discomfort, headache and stomach awareness [4]. Furthermore, the VR devices require a high-end computer to run [20]. The total cost to run the VR is higher than other traditional entertainment computer devices. Therefore, both the medical issue and high cost limits the VR growing speed into the consuming market.

\section{B. Argument Reality}

Argument Reality (AR) is a technical field that system reinforces the perception and knowledge of a real environment [2]. For instance, when the user turns on their smartphone's

\footnotetext{
* Corresponding author
} 
camera, the additional digital information relating to the content of camera capture will show on the screen. AR has three major characteristics: combination, real-time and interactive. Compared to VR, AR does not create any VE for the user to interact with. It instead adds a layer of information to the real environment [19]. Therefore, AR is a combination of the real world and the virtual which specific in visualize information. AR's second feature is much similar to VR because they share similar algorithms and technologies in many aspects [2]. The third feature has some small differences between AR and VR. VR focusses on the capturing of motion and manipulating this in the VE. AR pays more attention to collection of information from reality and transferring it to the system and provide cooperating elements to assist the user to interact with the real environment.

AR also has many bright prospects in some fields such as driving assistance, tourism, professional gesture assistance and game industry [2], [21], [22]. AR can use its combination of features to improve communication efficiency and provide visual information to better understand certain complex phenomena. However, AR has an inevitable contradiction. Although AR will not produce any discomfort sickness like VR, AR cannot provide the same immersive VR experience because AR is based on the reality and cannot generate the isolated VE. Furthermore, most of the AR devices are mobile phones and pads which have a limited screen size. The limited size and experience is the major weakness for AR.

\section{Mixed Reality}

Mixed Reality (MR) is a combination concept between AR and VR [3]. Fig. 1 illustrates the comparison between AR, MR and VR. Traditional VR generates a simulated virtual world with isolation from the real world, but MR can build an imagined environment under the realistic. Furthermore, MR and VR are using the head-mounted display (HMD) as the operating devices. Therefore, it implies that MR is a kind of VR in a broader sense. Traditional AR can build interactive virtual elements based on a realistic environment. However, AR, especially mobile AR cannot simulate a virtual world without practical environmental support. At the same time, MR can generate it as VR and also provide the interactive feature with reality. Compared to AR, MR more tends to virtual than reality. Considered the above information, it can conclude that MR moves much further than AR and VR by combining both of their advantages.

MR has greatly influenced the way people interact with information in the digital age, as such HoloLens provide the immersive user experience to explore the information with HoloLens' software support [1]. MR focuses on the medical and engineering industry because they require the users to mix the virtual environment and reality together to communicate and understand the information. Although MR combines the advantages of AR and VR, it still has some limitations. Firstly, the HMD of MR is very expensive due to its hardware cost. The high-cost limits the related market size. Secondly, MR has a narrow field of view [5]. Fig. 2 displays a comparison between the trailer and the released product. The trailer image shows that the user should have an immersive experience while

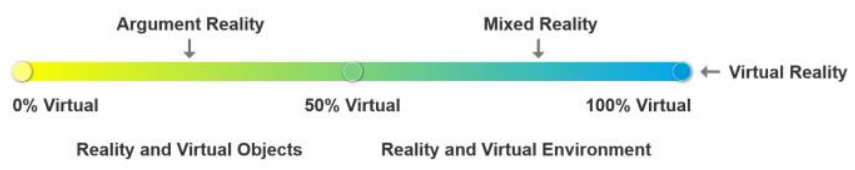

Fig. 1. Comparison between AR, MR and VR.

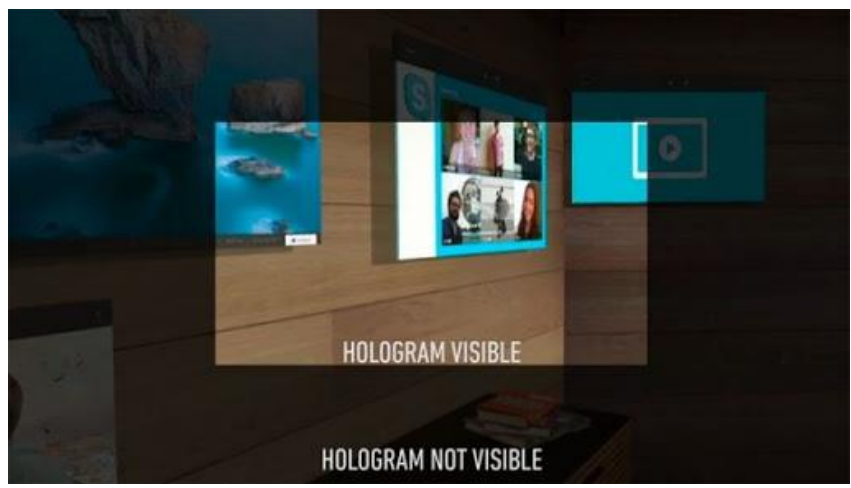

Fig. 2. HoloLens Field of View.

<Source: https://lh3.googleusercontent.com/>

using HoloLens. However, the released product only provides a limited view of the field which significantly affects the user experience. In a word, MR still has far more distance to widespread the consuming market.

\section{XR HISTORY AND TREND}

The previous section introduces the XR (VR, AR and MR) concept. Although different classified reality technologies have different goals, they still share similar technology to develop. For instance, Unreal Engine and Unity 3D are the most common developing kit in XR area [2]. Therefore, it is necessary to research the XR history and analyze how the XR concept starts and how XR divides into VR, AR and MR.

The following content divides XR history into two sections: Art History and Technical History. The reason for our research to discuss Art History is that XR concept was generated by the artist before the technical theory created. XR experience was not an exclusive concept in the science area. Many artists and writers have already used XR concept in their products as the fruit of pure fantasy even some scientist are inspired from the products. Consequently, both art and technical history should be reviewed so that it can better conclude the XR history and understand the developing trend for technology.

\section{A. Art History}

Fig. 3 concludes the XR history in the art and technical field. This section starts with the art aspect. In the 15th century, an Italian engineer Giovani Fontana designed an immersive system called CAVE which uses large lanterns to project the images into the walls of room [2]. The system was applied to displaying the artworks. This invention is the first time to point out the immersive and VR concept.

In the next few hundred years, more artists and engineers contributed to building the immersive concept. Antonin defines 


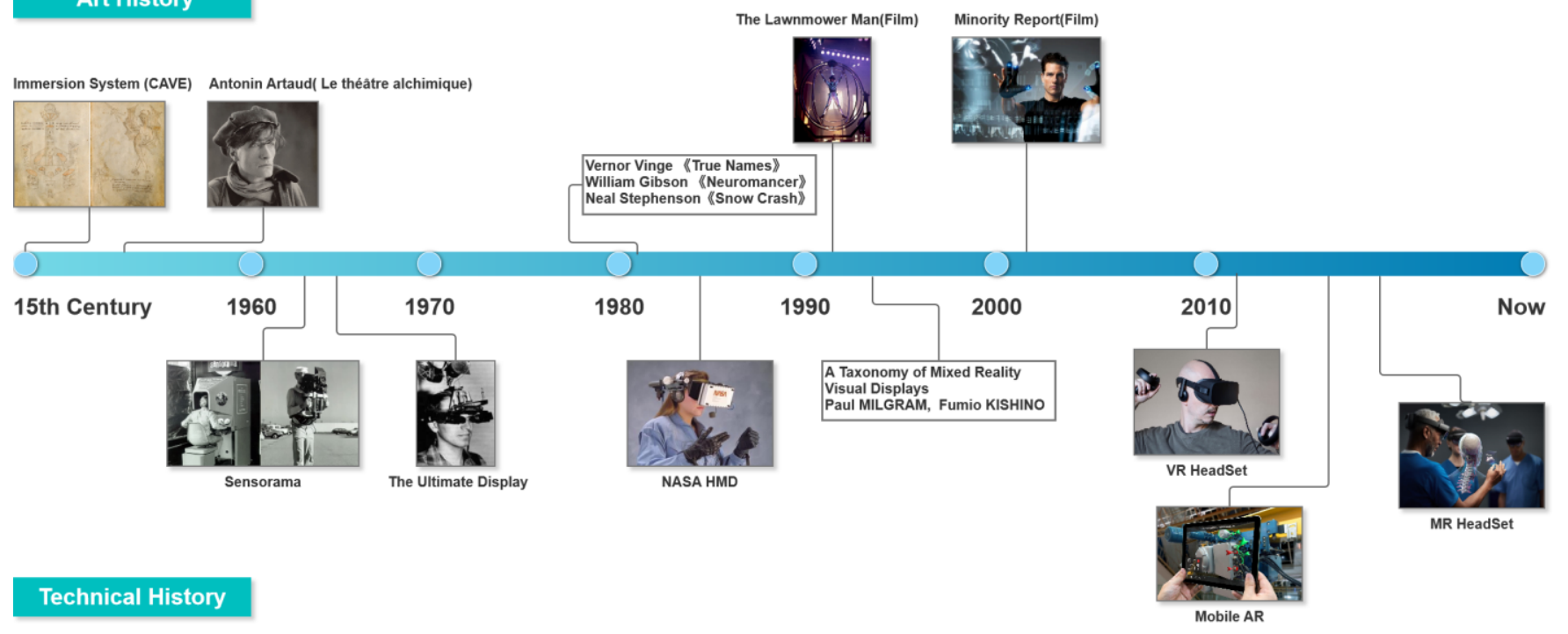

Fig. 3. XR History.

the modern concept of VR in 1958. Antonin is a famous inventor and artist in the Theatre area. He researched the elements in VR include characters, objects, images and so on [6]. His theory does not only beneficial for building the modern theater but also becomes the symbol of the evolution of the illusory world.

Science fictions also contributes to the development of the VR concept. From the 1980 s to the 1990 s, there are many science fiction media that describe an adventure story in a virtual world with future technology. 'True Names', 'Neuromancer' and 'Snow Crash' is the representee fiction in this stage [2]. Their books illustrate the different possible ways to interact with the virtual world like the online community concept and VR HMD. Although these concepts may have many differences in the present, they open the scientists' view to imagine the future.

In 1992, the movie 'The Lawnmower Man' also helped society know more about the VR concept [2]. In 2002, 'Minority Report' is released and it describes how the user interacts with the virtual user interface in the reality [7]. The movie broaden the XR concept from the virtual world to interact with reality.

\section{B. Technical History}

The technical history section is shown in Fig. 3. In 1962, Heilig developed the machine 'Sensorama' to provide video playing with the immersive experience through stereoscopic graphics and audio [8]. Heilig was inspired by Antonin's virtual theater concept and wanted to design an "Experience Theater" [8]. Sensorama uses Antonin's VR framework to design a brand new methodology to interact with the virtual world. It is one of the earliest VR systems.

However, Sensorama is a vast machine and cannot be portable. Therefore, the advanced portable device called "The Ultimate Display“ was invented by Robert Sproull and Ivan Sutherland in 1965 [9]. The Ultimate Display is widely considered as the first HMD in the VR industry [9]. They connected together VR and portability, so the HMD concept is generated. In the next following decades, scientists and corporations developed their VR device based on the HMD foundation.

In 1984, NASA's Ames Research Center designed the HMD system called 'The Virtual Environment Workstation' [10]. Compared to the 'The Ultimate Display', NASA's HMD provides more powerful computer graphics and tracked the user's head-position and finger gestures [10]. The Virtual Environment Workstation defines the interactive methodology in VR such as head movement and gesture distinction.

With computer graphics upgrading, the HMD research area is not satisfied with exploring in the virtual world and starting to interact with reality. Milgram and Kishino put forward the 'Reality to Virtual' concept and redefined the VR in 1994 [11]. Furthermore, they summarize the industry development history and generate the idea of VR, AR and MR [11]. In the following years, the industry uses XR as the abstract concept of using HMD technology and dividing it into VR, AR and MR by different virtual ratios [2].

After 2010, XR technology starts to widespread in the commercial market. The first famous commercial VR device Oculus Rift, was launched in 2013 [2]. HTC Vive and Valve Index were released in the next following years [24]. AR technology focuses on mobile platforms such as Android and IOS in 2014 [2]. ARKit and ARCore become the major AR development framework in the mobile platforms [23]. In 2015, the MR device, HoloLens was released [2]. Significantly, XR is accessible to the public from 2010 to 2020.

\section{History Analysis}

Considered the above historical line, it is obvious that both art and technology in XR are complementary. VR concepts and the original framework can be said to have been born in art. However, the art idea may far away from technical 
circumstances. Therefore, the scientist needs to constantly improve VR devices. Sensorama, The Ultimate Display, NASA's HMD and Oculus Rift show that the VR industry goes through a gradual process to improve their product from defective to mature.

VR technical development also affects the art aspect, especially in science fiction and films. The writers started to generate the interactive concept which is inspired by NASA's interactive HMD. The interactive element brings VR to a wider area. The wider field usually needs to redefine the concept and new division. Milgram and Kishino conclude the VR, AR and MR by different levels of reality and use XR to summarize the VR, AR and MR.

The historical line shows that the conceptual idea efficiently benefits technical development. Therefore, it is necessary to analyze how the recent conceptual imagination has become reality. The following section uses the article 'Creating next generation blended learning environments using mixed reality, Video Games and Simulations' as a case study to analyze how the concept affects technologies.

\section{CASE STUDY}

Kirkley writes the 'Creating next generation blended learning environments using mixed reality, Video Games and Simulations' in 2005 [12]. The paper receives 269 citations by 25th December 2020. We use the article as a case study because it illustrates the future projections with high quality of theoretical analysis and receives high citation numbers. If there are any technical achievements in the XR educational area, most of them will refer to it. Therefore, using the citation date to track the XR education historical trend is more efficient.

Our research summarizes its major future views and analyzes which citation achieves the imagination in the technical field. Furthermore, we statistically analyze the citations of different papers based on their underlying area to understand the developing trends in the XR educational field. With critical analysis, we believe that the data can help us to have a better methodology of discussing the overall concept and imagination.

\section{A. Future Views with Citations}

Kirkley builds up the MR education conceptual framework and illustrates the possible views in the future [12]. She divides them into two sections: learning environments and training technologies. Learning environments focus on how to build the virtual world. Training technologies pay more attention to the interaction between virtual and reality. We summarize her expectations as follows.

1) Learning environments: Educators can use the simulation to show the history onsite and watching a digital reenactment of a battle in real-time [12].

2) Learning environments: In the training simulation, military soldiers can use the HMD to receive the indicated information of any co-existing object in VE such as vehicles, buildings and friends fire [12].
3) Training technologies: Students use a handheld computer to get the location-based information in the outdoor activity of environmental science course [12].

4) Training technologies: Geology researchers can use the HMD to interact with geology by digital notes instead of studying in the research lab [12].

5) Training technologies: Architectural students can project the virtual CAD (Computer-aided Design and Drafting) model into the real plastic model of a city so they can examine how different types of buildings interact with reality [12].

The first Kirkley's expectation focuses on building the virtual environment. In 2012, Garrett went on to develop a learning platform [13]. The platform assists the educator to use the 3D game technology to train in the mining industry [13]. Fig. 4 is a system image. The project successfully achieves Kirkley's first expectation which generates the simulation environment to educate the students and workers in the related fields.

From 2010, there are many military training simulation systems released into the military industry. Variable Priority Training (VPT), Simulation Based Training (SBT), Simulation-based Team Training (SBTT) and Demonstrationbased Learning (DBT) are the major training systems in the air force industry [14]. Both systems design a comprehensive environment to ask the operator to meet with the Unmanned Aerial Systems (UAS) criteria. Considered above military products, the Kirkley's military expectation has been achieved in 2010.

Santoso, Yan and Gook developed an AR application to assist students' spatial cognitive ability in 2012 [15]. The application was developed on the iPad platform and used the camera to track the marker object in reality and display the $3 \mathrm{D}$ virtual elements beside the marker object [15]. The project requires users to point the colorful AR marker on the book so the system will display the digital information [15]. The project uses mobile devices to develop the educational purpose of AR systems. The project achieves the three central concepts of the third expectation, including 'Handed Computer', 'Locationbased Information' and 'Science Course'.

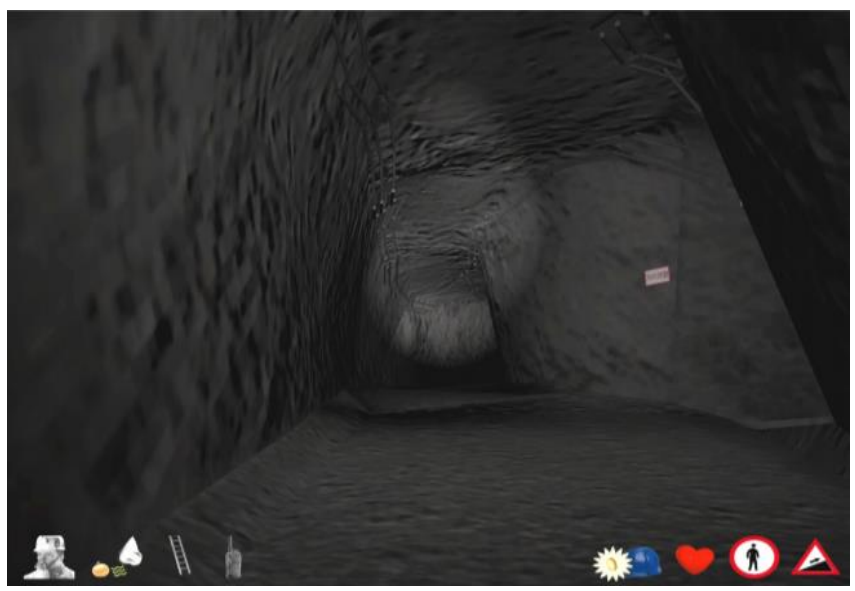

Fig. 4. Servicing infrastructure within the virtual mine [13]. 


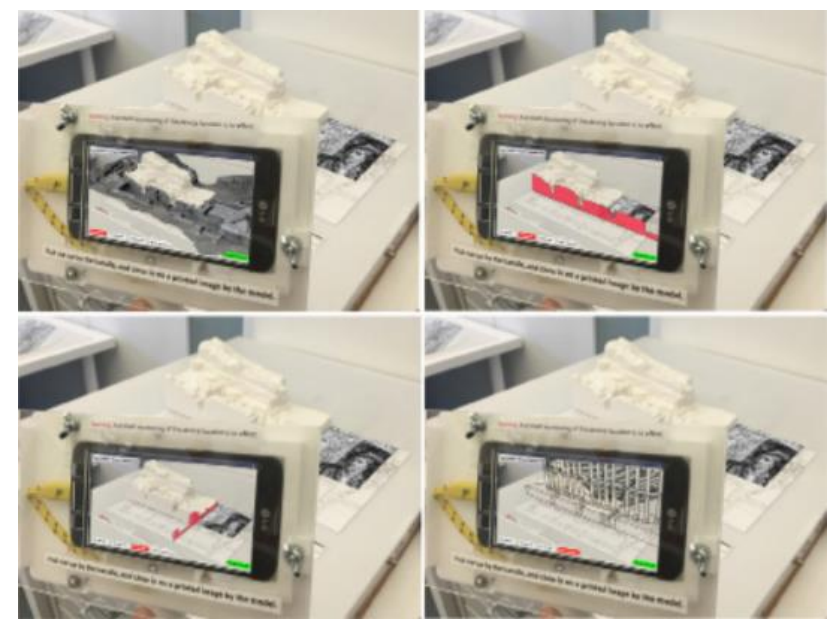

Fig. 5. How Multirama work [17].

NASA's OnSight system was developed to use 3D captured images to build a 3D virtual Mars environment [17]. The VE uses HoloLens, an MR device to display with stereoscopic [17]. While multi researchers wear HoloLens in the same space, they can cooperate to interact with the virtual environment [17]. It is much more efficient than research from books or computer screens. It is significant that NASA's project successfully achieves the fourth expectation.

ARC Group of MIT first developed Multirama in 2013 and improved on the architecture usage in 2015 [17]. Fig. 5 illustrates that the project uses solid models as raw data and adds the digital model and information by mobile devices as an overlay [17]. The project also provides interactive functions to help the architecture students better understand the relationship between the real and the virtual world. There is no doubt that the fifth expectation has become a reality.

By analyzing the above citations, it is significant that Kirkley's expectations have been achieved. Each of the first achievements where developed in the same period, from 2010 to 2015. Therefore, it is necessary to discuss why the application achievements are born at the same time and the process of how the theory becomes the application.

\section{B. How to Achieve the Expectations?}

To discuss how the theory becomes the application, we extracted 269 citations and analyzed the data. We divided the citations into three sections: Theory, Application and Unknown.

1) Theory: Theory concept includes theoretical concepts, conceptual frameworks, literature review and social investigation.

2) Application: Application concept includes algorithm, application, system structure and interactive development.

3) Unknown: Unknown paper means the paper is not written in English and has no English title and abstract. In order to avoid any mistake by translation, we will place these articles into the Unknown group.

Table.1 illustrates each year's citation situation.
TABLE I. Citation STATISTIC

\begin{tabular}{|c|c|c|c|}
\hline Year & Theory & Application & Unknown \\
\hline 2005 & 3 & 0 & 0 \\
\hline 2006 & 10 & 1 & 0 \\
\hline 2007 & 6 & 1 & 0 \\
\hline 2008 & 13 & 3 & 1 \\
\hline 2009 & 8 & 2 & 0 \\
\hline 2010 & 11 & 4 & 3 \\
\hline 2011 & 23 & 4 & 0 \\
\hline 2012 & 14 & 4 & 2 \\
\hline 2013 & 12 & 3 & 3 \\
\hline 2014 & 14 & 10 & 0 \\
\hline 2015 & 5 & 3 & 0 \\
\hline 2016 & 9 & 8 & 2 \\
\hline 2017 & 17 & 8 & 2 \\
\hline 2018 & 5 & 12 & 3 \\
\hline 2019 & 11 & 13 & 2 \\
\hline 2020 ( Untill 25th & 5 & 9 & 0 \\
\hline December 2020) & 5 & & \\
\hline
\end{tabular}

Based on our data collection, we can summarize some significant trends. We divide the years into four periods.

The first period is from 2005 to 2007. The period has no significant application papers relating to XR. Although two articles can conclude the application, both articles focus on illustrating the web design and 2D game design in the educational field. Fig. 6 displays how the application looks in the first period.

The second period is from 2008 to 2013 . The period has some application papers relating to XR but the application paper appears less than $50 \%$ in the analysis compared to the theory papers. In this period, the researchers and developers have designed the immersive VE with 3D technology from game industry. Therefore, Kirkley's learning environmental expectations have been achieved at this stage. However, this period focuses on the VE which is the VR field. Although there are some inventions from the AR area such as Santoso's project, it still has limitations in the AR and MR areas.

The third period is from 2014 to 2017. The period has many application papers relating to XR with the application papers appearing more than $50 \%$ of the time compared to the theory papers. Compared to the second period, the third period generates much more interactive projects in AR and MR area. Kirkley's learning technologies expectations have entirely become reality in this period.

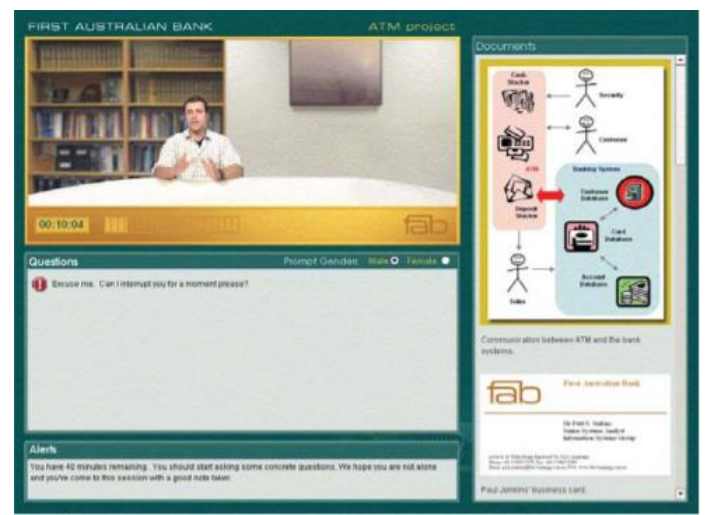

Fig. 6. Application paper in first perioid [18]. 
The fourth period is from 2018 to 2020 . This period has far more application papers relating to XR. Furthermore, the application papers have more than theory papers for the first time. Due to the Internet service quality improves allowing high-speed information transmission, many researchers start to look at the XR technology to improve virtual learning.

Considering both periods' data, it is significant that the XR research area has a complementary relationship with the XR industry. Before the technical breakout, the technology limited most of the researchers' invented space. The educational researchers only can start from theoretical orientation. After computer graphics technology rapidly raised, the VE concept was achieved in education applications. After the commercial $\mathrm{XR}$ devices have been released, interactive technology can be applied to the educational area. Based on the data, we can see how technology gradually improves the educational technology in the XR area.

\section{Keyword Trending Analysis}

The previous section illustrates the gradual developing flow in the educational XR field. It divides the timeline into four sections and explains different period's characteristics. However, the fourth period is too short to summarize its significant trend. It is necessary to discuss the future development path. Consequently, our research uses the data analysis software to analyze all the citation papers' abstracts. We try to find the keyword relationship and trend in different stages in the statistical software. Some of the words needed to be filtered. In the following section, we will set our filter rule and explain how the data analysis works.

We collected the filter word by the following rule. The first rule was filtering the XR topic based on whether the keyword in the article's topic is VR or AR. The second rule is filtering the educational keywords as education is the primary topic for our case study. Table. 2 illustrates the filtering word list.

TABLE II. FILTERING WORDLIST

\begin{tabular}{|c|c|}
\hline Rule & Words \\
\hline XR Topic & $\begin{array}{c}\text { Virtual Reality, VR, Argument Reality, Mixed } \\
\text { Reality, MR, Extended Reality, XR, Reality }\end{array}$ \\
\hline Education Topic & Learning, Education, Teaching, Student, \\
Study, Training
\end{tabular}

TABLE III. WORDLIST $\left(1^{\mathrm{ST}}, 2^{\mathrm{ND}}, 3^{\mathrm{RD}}\right.$ AND $4^{\mathrm{TH}}$ PERIOD $)$

\begin{tabular}{|c|c|c|c|c|}
\hline $\begin{array}{l}\text { Word (1 } \\
\text { Period) }\end{array}$ & $\begin{array}{l}\text { Word }\left(2^{\text {nd }}\right. \\
\text { Period })\end{array}$ & $\begin{array}{l}\text { Word }\left(3^{\text {rd }}\right. \\
\text { Period) }\end{array}$ & $\begin{array}{c}\text { Word (4th } \\
\text { Period) }\end{array}$ & Pos \\
\hline game & technology & technology & technology & $1 \mathrm{st}$ \\
\hline video & environment & environment & design & 2nd \\
\hline research & development & development & application & $3 \mathrm{rd}$ \\
\hline design & simulation & simulation & research & 4th \\
\hline technology & experience & application & development & 5 th \\
\hline tool & understanding & participants & model & 6th \\
\hline blend & participants & understanding & science & 7 th \\
\hline method & construction & literature & environment & 8th \\
\hline E-learning & performance & experience & blend & 9th \\
\hline literature & application & interactive & robot & 10th \\
\hline
\end{tabular}

We analyze the abstract's words from 269 citations. Some of the words have different forms like singular and plural. Therefore, we combine similar words and list them by different periods. Table. 3 displays the word list with rank.

From Table.3 data, we can summarize some significant trends.

Firstly, 'Technology' stays at the fifth position in the first period. It jumps to the first position in the second period and occupies the first position till the fourth period. Significantly, technology is a critical feature in the XR educational articles.

Secondly, 'Environment' plays the second important role in the second and third period. However, it drops its position to eighth in the present. Considered the previous research, VE is an important research field in the second period. However, the development trend may move to a new orientation in the fourth period. It no longer plays the same role.

Thirdly, 'Application' plays a more important role from the second to the fourth period. In the second period, 'Application' only stays in the last position. The keyword rises gradually and occupies the fifth position in the second period. In the fourth period, it jumps to the third position. There are more and more XR applications and projects due to the commercial XR devices being released. Consequently, the industry stimulates the research achievements' boom up.

Fourthly, we consider the keyword in the fourth period which has not appeared in the previous periods. 'Model', 'Science' and 'Robot' are the new keywords. In order to analyze better in the fourth period, we discuss the articles with related keywords. It was realized that XR moves to the science education and robot field in recent years. XR provides immersive and stereo experiences in studying and communication especially through the HoloLens (MR HMD). Furthermore, the XR gesture tracking can assist the scientist to improve how humans can control the robots.

By analyzing the above trends, we can summarize the possible future trend in the fourth period. 'Technology' continues its crucial feature. 'Application' plays a critical role instead of 'Environment'. Many science fields such as 'Robot' will use XR technology to open a new horizon in the XR industry.

\section{CONCLUSION}

This paper describes three classified XR technology concepts and illustrates their history by the technical and article timeline. By analyzing how the recent conceptual theory has become reality, we used 'Creating next generation blended learning environments using mixed reality, Video Games and Simulations' as a case study. At first, we summarized its expectations and reviewed the citations which achieved them. Furthermore, we divided the citations into four periods by the percentage of technical articles. We used our timeframe to discuss a keyword appearance list, to conclude the significant trends and put forward a possible expectation for future developments in the XR educational field.

Our research project contains the XR educational field and considered using Web XR technology to improve the online 
educational platform. Future work will extend our research field to discuss more XR user interface and Web XR approaches combined with industrial standards. We will analyze their working principles to improve our web XR research program.

\section{REFERENCES}

[1] Flavián C, Ibáñez-Sánchez S, Orús C. "The impact of virtual, augmented and mixed reality technologies on the customer experience," Journal of Business Research, 2019, 100: 547-560.

[2] Arnaldi, B., Guitton, P., \& Moreau, G. (Eds.) . "Virtual reality and augmented reality: Myths and realities," John Wiley \& Sons, 2018.

[3] Speicher M, Hall B D, Nebeling M. "What is mixed reality?" , Proceedings of the 2019 CHI Conference on Human Factors in Computing Systems. 2019: 1-15.

[4] Weißker T, Kunert A, Fröhlich B, et al. "Spatial updating and simulator sickness during steering and jumping in immersive virtual environments", 2018 IEEE Conference on Virtual Reality and 3D User Interfaces (VR). IEEE, 2018: 97-104.

[5] Kun A L, Meulen H, Janssen C P. "Calling while driving: An initial experiment with HoloLens". 2017.

[6] Artaud A. "Theatre and its Double", Alma Books, 2018.

[7] Dick P K. "Tiptree J. Minority report", London: Gollancz, 2002.

[8] Heilig M L. "Sensorama simulator: U.S. Patent 3,050,870”, 1962-8-28.

[9] Sutherland I E. "The ultimate display", Multimedia: From Wagner to virtual reality, 1965, 1.

[10] McGreevy M W. "The virtual environment display system”,1991.

[11] Milgram P, Kishino F. "A taxonomy of mixed reality visual displays" ,IEICE TRANSACTIONS on Information and Systems, 1994, 77(12): 1321-1329.

[12] Kirkley S E, Kirkley J R. "Creating next generation blended learning environments using mixed reality, video games and simulations", TechTrends, 2005, 49(3): 42-53.

[13] Garrett M. "Developing knowledge for real world problem scenarios: Using 3D gaming technology within a problem-based learning framework", 2012.
[14] Ison D C, Terwilliger B A, Vincenzi D A. "Designing simulation to meet UAS training needs", International Conference on Human Interface and the Management of Information. Springer, Berlin, Heidelberg, 2013: 585-595.

[15] Nincarean D, Alia M B, Halim N D A, et al. "Mobile Augmented Reality: the potential for education", Procedia-social and behavioral sciences, 2013, 103(0): 657-664.

[16] Noor A K. "The hololens revolution", Mechanical Engineering, 2016, 138(10): 30-35.

[17] Güleç Özer D, Nagakura T. "Vlavianos N. Augmented reality (AR) of historic environments: Representation of Parion Theater, Biga, Turkey", A|Z ITU Journal of the Faculty of Architecture, 2016, 13(2): 185-193.

[18] Cybulski J, Parker C, Segrave S. "Touch it, feel it and experience it: Developing professional IS skills using interview-style experiential simulations", ACIS 2006: Proceedings of the 17th Australasian Conference on Information Systems, Adelaide Convention Centre, 6th8th December 2006: thought leadership in IS. Australasian Conference on Information Systems, 2006.

[19] Ro, Young K., Alexander Brem, and Philipp A. Rauschnabel. "Augmented reality smart glasses: Definition, concepts and impact on firm value creation." Augmented reality and virtual reality. Springer, Cham, 2018, 169-181.

[20] Avila, Lisa, and Mike Bailey. "Virtual reality for the masses. "IEEE Annals of the History of Computing 2014, 34.05 : 103-104.

[21] Kimura R, Matsunaga N, Okajima H, et al. "Driving assistance system for welfare vehicle using virtual platoon control with augmented reality ", 2017 56th Annual Conference of the Society of Instrument and Control Engineers of Japan (SICE). IEEE, 2017, 980-985.

[22] Mgbemena, Chika Edith, et al. "Gesture detection towards real-time ergonomic analysis for intelligent automation assistance." Advances in Ergonomics of Manufacturing: Managing the Enterprise of the Future. Springer, Cham, 2016, 217-228.

[23] Nowacki, Paweł, and Marek Woda. "Capabilities of arcore and arkit platforms for $\mathrm{ar} / \mathrm{vr}$ applications." International Conference on Dependability and Complex Systems. Springer, Cham, 2019.

[24] Huang, Yilei, Samjhana Shakya, and Temitope Odeleye. "Comparing the functionality between virtual reality and mixed reality for architecture and construction uses." Journal of Civil Engineering and Architecture 2019, 13: 409-414. 